\section{A) Check for updates}

Cite this: Polym. Chem., 2019, 10 5645

Received 13th August 2019, Accepted 2nd October 2019

DOI: 10.1039/c9py01222b

rsc.li/polymers

\title{
Poly(Boc-acryloyl hydrazide): the importance of temperature and RAFT agent degradation on its preparation $\uparrow$
}

\author{
Oliver Creese, Pavan Adoni, Guanlong Su, Andrey Romanyuk (iD and \\ Paco Fernandez-Trillo (iD *
}

\begin{abstract}
Poly(acryloyl hydrazide) is a versatile polymer scaffold readily functionalised through post-polymerisation modification with aldehydes to yield polymers for biological applications. However, its polymerisation is affected by nucleophilic degradation of the RAFT agent that leads to early termination, an issue often overlooked in the polymerisation of primary acrylamides. Here we report the effect of temperature on the RAFT polymerisation of $N^{\prime}$-(tert-butoxycarbonyl)acryloyl hydrazide (1) and demonstrate that by carefully selecting this polymerisation temperature, a balance between rate of polymerisation and rate of degradation of the RAFT agent can be achieved. This way greater control over the polymerisation process is achieved, allowing the synthesis of Boc-protected poly(acryloyl hydrazide) with higher degrees of polymerisation (DP) than those obtained previously, while still maintaining low dispersities $\left(\Theta_{M}\right)$.
\end{abstract}

\section{Introduction}

Synthetic polymers are increasingly becoming an attractive means of interfacing biological systems via multivalent binding, displaying activities orders of magnitude higher than those of their monovalent components. ${ }^{1-5}$ Thus, polymers are now widely researched for biomedical applications including as antimicrobials, ${ }^{6,7}$ as drug and gene delivery vehicles, ${ }^{2,8}$ as biological sensors, ${ }^{9,10}$ or as "smart" biomaterials with antifouling properties. ${ }^{11}$ Highly functional polymers developed for specific applications generally involve the use of functional monomers which either already possess the final desired functionality, or have the capability of undergoing post-polymerisation modification to introduce the desired functionality. This latter approach can greatly broaden the scope of chemical functionalities used. Post-polymerisation modification has normally relied on click chemistries, ${ }^{12}$ and has now been greatly expanded through the use of oxime ${ }^{13}$ and hydrazone chemistry $^{14,15}$ reductive amination, ${ }^{16}$ and epoxide ring opening. ${ }^{17}$

A common limitation when developing synthetic polymers for biomedical applications is the need to screen large libraries of compounds which is costly and time consuming. In this regard, poly(acryloyl hydrazide) has been recently reported as a

School of Chemistry, and Institute of Microbiology and Infection, University of Birmingham, Edgbaston, B15 2TT Birmingham, UK.

E-mail:f.fernandez-trillo@bham.ac.uk

$\dagger$ Electronic supplementary information (ESI) available. See DOI: 10.1039/ c9py01222b versatile platform for the synthesis and screening of polymers for biomedical applications. ${ }^{14,18-20}$ Functional polymers are obtained by simple incubation of poly(acryloyl hydrazide) with functional aldehydes, both under aqueous or organic conditions, ${ }^{14}$ and this polymer has now been applied to the development of glycan arrays, ${ }^{18} \mathrm{pH}$ sensitive drug-delivery, ${ }^{21}$ and nucleic acid delivery. ${ }^{20,22,23}$ In our laboratories poly(acryloyl hydrazide) was prepared from Boc-protected precursor Boc- $\mathbf{P}_{\mathbf{x}}$ (Scheme 1) following deprotection with TFA. ${ }^{14}$ Reversible addition-fragmentation chain-transfer (RAFT) polymerisation of $N^{\prime}$-(tert-butoxycarbonyl) acryloyl hydrazide (1) resulted in a small library of polymers. However, control over the polymerisation was lost with increasing conversion and degree of polymerisation, possibly as a result of degradation of the RAFT agent through intramolecular nucleophilic attack. This degradation has been reported in the RAFT polymerisation of other acrylamide derivatives, ${ }^{24,25}$ including closely related methacryloyl hydrazide, ${ }^{26}$ with better control reported when the polymerisation is carried out at low temperatures. ${ }^{25,27}$ This sidereaction is often overlooked in the polymerisation of primary and secondary acryl- and methacrylamides, and makes synthesising highly functional polymers from this type of monomers inherently challenging. ${ }^{28}$ The need for greater control over these materials is more significant when looking to understand better the nature of the structure-activity relationship throughout post-polymerisation modification and biological screening.

Here, we report the effect of temperature and the decomposition rate of the initiator on the polymerisation of $N^{\prime}$-(tertbutoxycarbonyl)acryloyl hydrazide (1), as a route to optimise the preparation of poly(acryloyl hydrazide). Polymerisations 

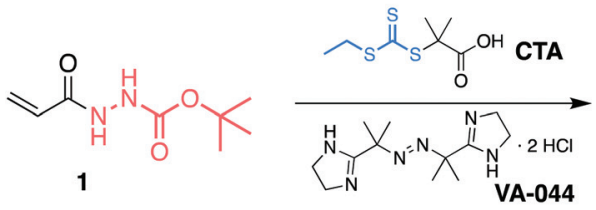

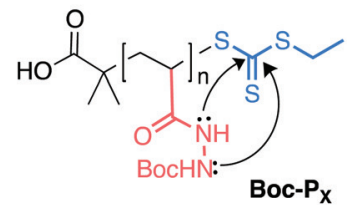

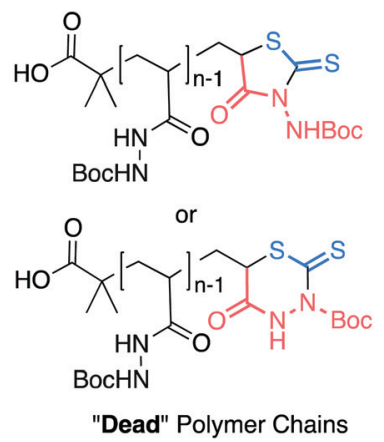

Scheme 1 RAFT polymerisation of $N^{\prime}$-(tert-butoxycarbonyl)acryloyl hydrazide (1) and potential degradation by-products.

were carried out using 2,2'-azobis[2-(2-imidazolin-2-yl) propane]dihydrochloride (VA-044) as a low temperature initiator, so that the rate of generation of radicals could be readily modified as a function of temperature. Our results suggest that while increasing the temperature increases the polymerisation rate, it also speeds up RAFT degradation and thus, loss of control. Conditions have been identified for which the polymerisation "outperforms" this side reaction and polymers with good control over molecular mass and dispersities $\left(\bigoplus_{M}\right)$ can be obtained. More importantly, these conditions allowed us to prepare Boc- $\mathbf{P}_{\mathbf{x}}$ with higher degrees of polymerisation and lower $\bigoplus_{\mathrm{M}}$, not accessible with our previous conditions. ${ }^{14}$ This improved control over the polymerisation of Boc-protected poly(acryloyl hydrazide) will be of value when DP and $\emptyset_{\mathrm{M}}$ may underpin future applications.

\section{Experimental section}

Materials

2-((Ethylthio)carbonothioyl)thio-2-methylpropanoic acid $(\text { CTA })^{29}$ and $N^{\prime}$-(tert-butoxycarbonyl)acryloyl hydrazide $(\mathbf{1})^{14,20}$ were synthesised according to protocols described in the literature. 2,2'-Azobis[2-(2-imidazolin-2-yl)propane] dihydrochloride (VA-044) was purchased from Fluorochem and used without further purification. All other chemicals were purchased from Sigma-Aldrich $₫$, Fisher Scientific $\AA$, VWR $₫$ or Acros $\AA$, and used without further purification. All solvents were Reagent grade or above, purchased from Sigma-Aldrich®, Fisher Scientific $®$ or VWR $®$, and used without further purification. Polymethylmethacrylate standards were purchased from Agilent®.

\section{Characterisation}

Nuclear Magnetic Resonance (NMR) spectra were recorded on either a Bruker Avance III $300 \mathrm{MHz}$ or a Bruker Avance III $400 \mathrm{MHz}$ spectrometer. Chemical shifts are reported in ppm (units) referenced to the following solvent signals: dimethylsulfoxide (DMSO)- $\mathrm{d}_{6} \mathrm{H}$ 2.50. Gel Permeation Chromatography (GPC) was performed with a Shimadzu Prominence LC-20A fitted with a Thermo Fisher Refractomax 521 Detector and a SPD20A UV-vis Detector. Poly( $N^{\prime}$-(tert-butoxycarbonyl)acryloyl hydrazide) (Boc- $\mathbf{P}_{\mathbf{x}}$ ) was analysed using $0.05 \mathrm{M} \mathrm{LiBr}$ in dimethylformamide (DMF) at $60^{\circ} \mathrm{C}$ as the eluent, and a flow rate of $1 \mathrm{~mL} \mathrm{~min}^{-1}$. The instrument was fitted with a Polymer Labs PolarGel guard column $(50 \times 7.5 \mathrm{~mm}, 5 \mu \mathrm{m})$ followed by two PLGel PL1110-6540 columns $(300 \times 7.5 \mathrm{~mm}, 5 \mu \mathrm{m})$. Molecular masses were calculated based on a standard calibration method using polymethylmethacrylate standards.

\section{RAFT polymerisation of $N^{\prime}$-(tert-butoxycarbonyl)acryloyl hydrazide (1)}

In a typical kinetic experiment 2,2'-azobis[2-(2-imidazolin-2-yl) propane]dihydrochloride (VA-044) (11.7 mg, $0.036 \mathrm{mmol}$ ), 2-ethylthiocarbonothioylthio-2-methylpropanoic-acid (CTA) (40.3 $\mathrm{mg}, 0.18 \mathrm{mmol}$ ) and $N^{\prime}$-(tert-butoxycarbonyl)acryloyl hydrazide (1) (1.666 g, $8.950 \mathrm{mmol}$ ) were dissolved in DMSO $(10.0 \mathrm{~mL})$ and a $100 \mu \mathrm{L}$ sample was taken at this stage to calculate conversion $(\rho)$. The solution vessel was sealed with a septum, securely fastened with electrical tape to maintain the seal, and degassed by bubbling with argon for 25 minutes. Using a cannula, $1 \mathrm{~mL}$ of the solution was transferred to sealed glass vials containing stirrer bars, each degassed for 5 minutes. Vials were then left to react at a pre-set temperature $\left(30-150^{\circ} \mathrm{C}\right)$ for the required amount of time. The reaction was stopped by allowing the tube to cool using a water bath and exposing it to air. $100 \mu \mathrm{L}$ aliquots of each timepoint were taken at this stage to calculate conversion $(\rho)$ and for GPC analysis. NMR and GPC analysis of each timepoint was carried out from the crude mixture. The natural logarithm of the inverse of the fractional concentration of monomer $-\ln \left(M_{0} / M_{\mathrm{t}}\right)$ - was plotted against time, and the data fitted using GraphPad Prism version 6.0 for Mac Os X, GraphPad Software, La Jolla California USA, http://www.graphpad.com. The in-built segmental line regression was used to fit the data to two intersecting lines. This model was used to identify when a change in the polymerisation kinetics was observed $\left(t_{\text {dead }}\right)$.

\section{Results and discussion}

As reported, our initial efforts to optimise the polymerisation of Boc-protected acryloyl hydrazide 1 focused on reducing the temperature of the polymerisation. ${ }^{14}$ RAFT polymerisation of 
acrylamides and methacrylamides often suffers from cleavage of the RAFT agent through intramolecular addition-elimination of the weakly nucleophilic amides to the trithiocarbonate group (Scheme 1). ${ }^{25}$ Under our previously reported conditions for the polymerisation of $\mathbf{1}$, a change in the rate of polymerisation was observed with increasing conversion (Fig. S1A $\dagger$ ) which we associated with this degradation of the terminal trithiocarbonate in the growing chain. It has been proposed that reducing the polymerisation temperature would significantly reduce the rate of this side reaction..$^{25}$ Thus, optimisation of the polymerisation was at that time carried out under the same conditions but using initiators with different 10 hours half-life decomposition temperatures $\left(t_{10}\right)$ (Fig. S1 $\dagger$ ). This way, the rate of formation of radicals was kept as similar as possible for all polymerisations while reducing the temperatures to $50{ }^{\circ} \mathrm{C}(\mathbf{V}-65)$ or $44{ }^{\circ} \mathrm{C}(\mathbf{V A - 0 4 4})$. Despite the use of lower temperatures, in all cases, a change in the kinetics of the polymerisation was observed, although this change was not as obvious for the polymerisations performed at $44{ }^{\circ} \mathrm{C}$ (Fig. S1A, $\uparrow$ right). To identify when this change in rate of polymerisation was occurring, the natural logarithm of the inverse of the fractional concentration of monomer $-\ln \left(M_{0} / M_{\mathrm{t}}\right)$ - was plotted against time, and the data fitted to a segmental line regression. This function fits the data to two different lines, before and after a breakpoint. In our case, we termed the breakpoint $t_{\text {dead }}$ because we think that after this point, side reactions have a predominant effect on the kinetics of the polymerisation resulting in an increasing number of dead polymer chains. This change in kinetics was reflected on the relatively high dispersity in molecular mass $\left(D_{\mathrm{M}}=1.38-1.95\right)$ obtained for the polymers prepared under these conditions. ${ }^{14}$ Overall, no clear benefit from reducing the temperature was observed, with a $t_{\text {dead }}$ of approximately 4 and 4.5 hours for polymerisations at $50{ }^{\circ} \mathrm{C}$ and $70{ }^{\circ} \mathrm{C}$ respectively. Interestingly, $t_{\text {dead }}$ for the polymerisation performed at $44{ }^{\circ} \mathrm{C}$ was observed at approximately $2.5 \mathrm{~h}$, which would suggest degradation was occurring faster at this temperature. This was not expected and may suggest that other mechanisms beyond the simple degradation of the RAFT agent may be at play. For instance, polymerisation decay can also be caused by diminishing initiator efficiency at high monomer conversions, which has been observed for some azo-initiators. ${ }^{30,31}$ However, this factor normally becomes significant at much higher conversions than the ones we reported.

Attempts to perform the polymerisation at an even lower temperature $\left(30^{\circ} \mathrm{C}\right)$ using VA-044 as the source of radicals resulted in a very long induction period followed by a short period of linear increase of the fractional concentration of monomer until a change in kinetics was again evident (Fig. S2 $\dagger$ ). The maximum conversion in this case was $50 \%-\ln$ $\left(M_{0} / M_{\mathrm{t}}\right)=0.83$, worse than that observed for the polymerisations performed at higher temperatures.

In order to determine if degradation of the RAFT agent was indeed possible at low temperatures, we attempted to synthesise a small molecule analogue which mimicked an $n=1$ polymer (Scheme S1 $\dagger$ ). To this end, 2-bromopropionic acid (2) was reacted with tert-butyl carbazate, and the resulting bromine derivative 3 reacted under standard conditions for the formation of the RAFT agent. ${ }^{1} \mathrm{H}$ NMR analysis of this reaction revealed a very complex mixture, where only traces of something that could resemble trithiocarbonate 4 could be identified (Fig. S4 $\uparrow$ ). This observation was in line with our previous results, and suggested that hydrazide containing trithiocarbonates such as $\mathbf{4}$ were very amenable to intramolecular nucleophilic attack. Attempts to isolate this trithiocarbonate $\mathbf{4}$ were unsuccessful, with the main isolated product of this reaction being tentatively assigned to a mixture of the 5- and 6-membered rings in a $6: 4$ ratio (Fig. $55 \dagger$ ).

Seeing how lowering the temperature had no beneficial effect on the kinetics of the polymerisation of $\mathbf{1}$, and a change in kinetics was still observed, we decided to explore the use of "Ultra-Fast" polymerisation conditions in an attempt to outrun the side reaction..$^{32-34}$ Our hypothesis was that by using a low temperature initiator such as VA-044 at a significantly higher temperature $\left(\right.$ e.g. $\left.100{ }^{\circ} \mathrm{C}\right)$ than the reported $t_{10}\left(44^{\circ} \mathrm{C}\right)$, an increase in the concentration of radicals in solution would be achieved. This way, the concentration of propagating radicals would be higher with a greater number of chains growing at the same time, resulting in the synthesis of polymers with better control over the molecular mass and $D_{\mathrm{M}}$. This methodology is particularly suitable for fast-propagating monomers such as acrylamides, and since the rate of polymerisation is directly proportional to the concentration of these propagating radicals (and the monomer concentration, $R_{\mathrm{p}}=k_{\mathrm{p}}[\mathrm{M}]\left[\mathrm{P}^{*}\right]$ ), we postulated that running the polymerisation under these conditions could outperform the side reaction observed under standard RAFT polymerisation conditions. In a first attempt, the polymerisation conditions previously reported by us for the polymerisation of 1 (Fig. S1†) $)^{14}$ were modified so that the initiator used was VA-044 and the polymerisation temperature was $100{ }^{\circ} \mathrm{C}$. A shorter polymer was targeted this time and, as expected, the polymerisation was very fast, reaching up to $70 \%$ conversion in less than five minutes (Fig. 1A, CTA: VA-044 5:1 $\bullet$ ). The change in reaction rate could not be suppressed and was again evident, with a $t_{\text {dead }}$ of approximately $4.5 \mathrm{~min}$. Before $t_{\text {dead }}$, the polymerisation retained the features of a controlled polymerisation, with the molecular mass of the polymer directly proportional to the conversion and, comparable dispersities (Fig. 1B, left) to those observed with our previous conditions. ${ }^{14}$

These results were promising and we therefore explored decreasing the concentration of initiator in our polymerisations, in an attempt to suppress termination, increase the number of chains growing from the RAFT agent and thus optimising the dispersities. However, while dispersities were decreased, reducing the concentration of initiator in these polymerisations resulted in slower reactions, with no effect observed in $t_{\text {dead }}$ (Fig. 1A). As a result, the maximum conversion obtained when the CTA: VA-044 ratio was increased to $10: 1$ or $15: 1$ ( $40 \%$ and $24 \%$ conversion respectively) was lower than in the previous case (70\%).

We decided next to run the polymerisations at $150^{\circ} \mathrm{C}$, in an attempt to further increase the concentration of radicals 

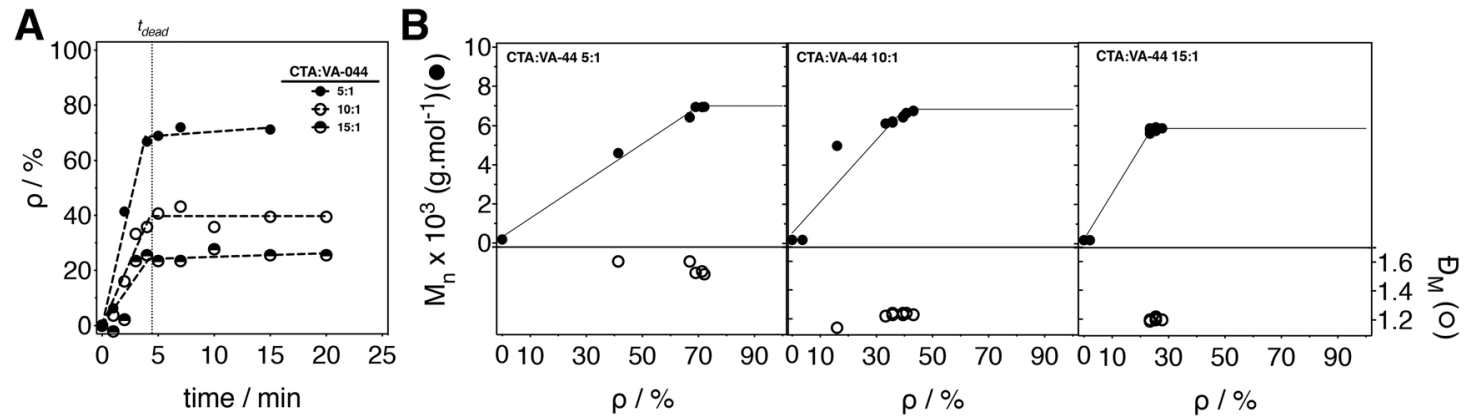

Fig. 1 (A) Plot of conversion ( $\rho$ ) vs. time and (B) measured number average molecular mass $\left(M_{n}\right)$ vs. conversion ( $(\bullet)$ and dispersity in molecular mass $\left(\Xi_{M}\right)$ vs. conversion $(O)$, for polymerisations of $N^{\prime}$-(tert-butoxycarbonyl)acryloyl hydrazide (1) performed with different CTA: VA-044 ratios. Conditions: $[\mathrm{M}]=0.9 \mathrm{M},[\mathrm{M}] /[\mathrm{CTA}]=50 / 1 . \mathrm{M}_{\mathrm{n}}$ and $\Xi_{\mathrm{M}}$ calculated by $\mathrm{GPC}$ using $0.05 \mathrm{M} \mathrm{LiBr}$ in dimethylformamide (DMF) at $60^{\circ} \mathrm{C}$.

during early stages of polymerisation, and thus the rate of propagation. However, these conditions not only resulted in lower conversions (Fig. S9†) but a colour change of the reaction mixture from yellow to dark brown, suggesting that thermal decomposition of the trithiocarbonate group was ocurring. ${ }^{35}$ Thermal decomposition of the RAFT agent was confirmed via ${ }^{1} \mathrm{H}$ NMR where signals consistent with the $\beta$-elimination of the trithiocarbonate could be observed (Fig. S10 †). ${ }^{35,36}$
Having identified conditions to run the polymerisation of $\mathbf{1}$ at $100{ }^{\circ} \mathrm{C}$, which resulted in similar conversions and dispersities to those previously reported, we decided to explore the use of these conditions to prepare polymers of higher DP (Fig. 2), which were harder to control using our previously reported method. ${ }^{14}$ Three different DPs were targeted (i.e. $[\mathbf{1}] /[\mathbf{C T A}]=$ 50,100 and 150), by maintaining the concentration of 1 and reducing the amount of RAFT agent and initiator used. As
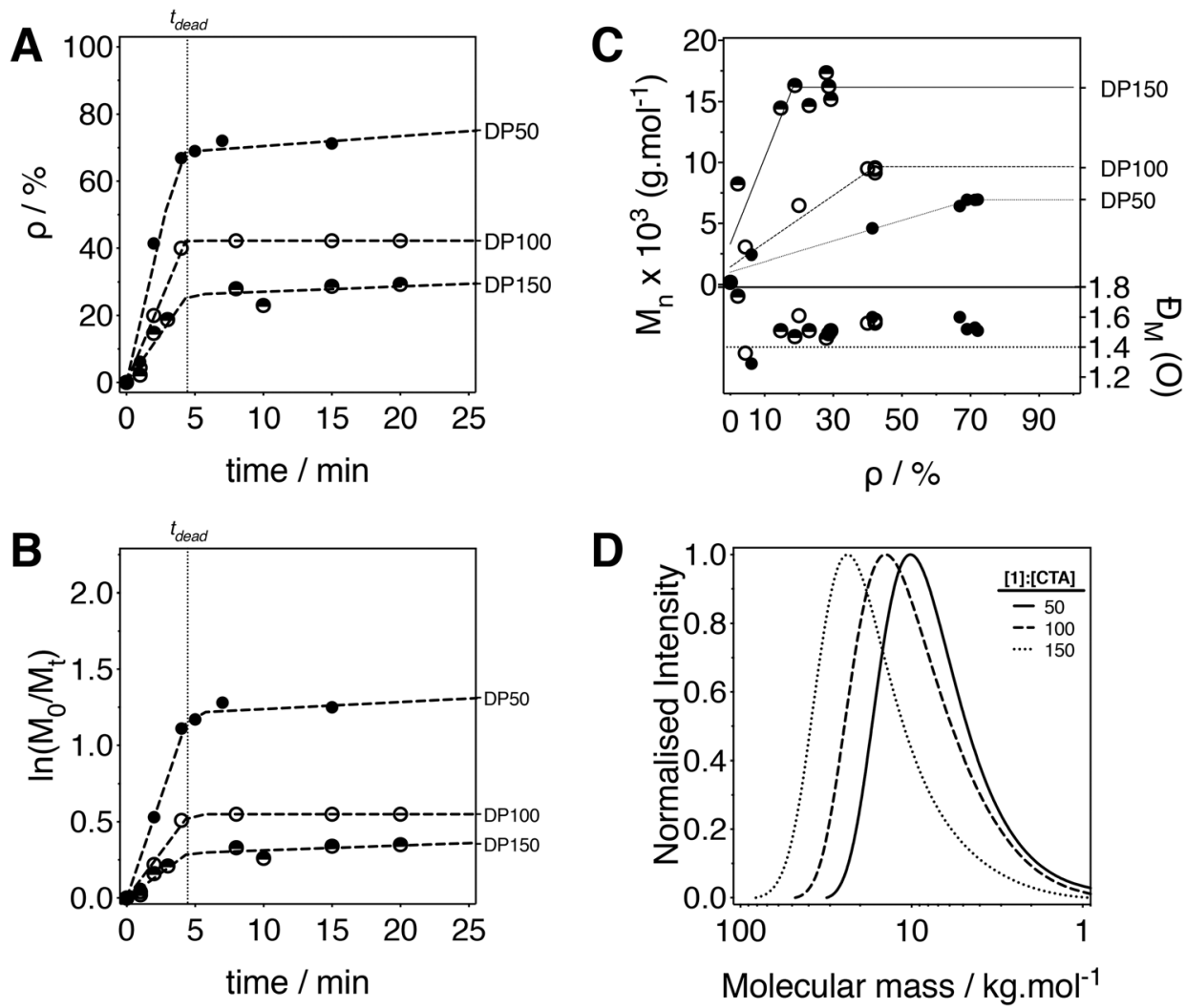

Fig. 2 (A) Plot of conversion ( $\rho)$ vs. time, (B) fractional concentration of monomer $\ln \left(M_{0} / M_{t}\right)$ vs. time, and (C) measured number average molecular mass $\left(M_{n}\right)$ vs. conversion $(\rho)$ (top) and dispersity in molecular mass $\left(\Theta_{M}\right)$ vs. conversion $(\rho)$ (bottom), for polymerisations of $N^{\prime}$-(tert-butoxycarbonyl) acryloyl hydrazide (1) performed at $100^{\circ} \mathrm{C}$ with different 1 : CTA ratios. (D) GPC chromatograms of the resulting polymers at the highest conversion obtained. Conditions: $[\mathrm{M}]=0.9 \mathrm{M},[\mathrm{CTA}] /[\mathrm{VA}-044]=5 / 1 . \mathrm{M}_{\mathrm{n}}$ and $\Xi_{\mathrm{M}}$ calculated by GPC using $0.05 \mathrm{M} \mathrm{LiBr}$ in dimethylformamide (DMF) at $60^{\circ} \mathrm{C}$. 
expected, this resulted in slower polymerisations, while $t_{\mathrm{dead}}$ still remained at around $4.5 \mathrm{~min}$ (Fig. 2A). As a consequence, polymerisations targeting 100 and 150 monomer units only reached low conversions ( $\sim 40 \%$ and $30 \%$ respectively). In any case, control over the molecular mass of the polymer was still observed during the first stages of the polymerisation, with the average molecular mass $\left(M_{\mathrm{n}}\right)$ increasing linearly with time until the change in polymerisation rate was evident $\left(t_{\text {dead }}\right)$ (Fig. 2B). A clear shift towards lower retention time was observed in the gel permeation chromatograms when higher DPs were targeted, suggesting that, at least during the initial phase of the reaction, the polymerisation was maintaining features of a controlled radical polymerisation. $D_{M}$ remained similar across the three targeted molecular masses which demonstrates an improvement compared to our previous conditions where $\emptyset_{M}$ increased with increasing targeted DP.

At this point, our results suggested that a compromise could be obtained between increasing the rate of propagation by increasing the polymerisation temperature, and delaying $t_{\text {dead }}$ by reducing the polymerisation temperature. Therefore, we investigated polymerisations at intermediate temperatures (Fig. 3). While a change in polymerisation rate was still evident for the new temperatures investigated, higher conversions could be achieved for the polymerisation performed at $65{ }^{\circ} \mathrm{C}$ $(90 \%)$ while the next highest conversions at $80^{\circ} \mathrm{C}$ and $50{ }^{\circ} \mathrm{C}$ were $77 \%$ and $80 \%$ respectively (Fig. 3B, 9 ). Temperature had a significant effect on the time at which a change in polymerisation rate was evident $\left(t_{\text {dead }}\right)$, with this inflection point happening sooner as the temperature was increased (Fig. 3B, O).

With encouraging results from the polymerisations at $65{ }^{\circ} \mathrm{C}$, we set out to probe the "livingness" of the polymer before and after $t_{\text {dead }}$ and thus whether $t_{\text {dead }}$ was due to degradation of the RAFT agent. To this end, we isolated and purified two polymerisations of $\mathbf{1}$, one that had been stopped at intermediate conversions ( $\rho=47 \%, t=30 \mathrm{~min}$ ), before $t_{\mathrm{dead}}$ (Fig. S6A $\dagger$ ) and one that was stopped at maximum conversion $(\rho=85 \%, t=120 \mathrm{~min})$, after $t_{\text {dead }}$ (Fig. S6B $\dagger$ ). As expected, Boc- $\mathbf{P}_{\mathbf{x}}$ isolated before $t_{\text {dead }}$ was able to undergo complete chain extension with further addition of $\mathbf{1}$ and initiator (Fig. S6A $\dagger$ ), thus demonstrating that at intermediate conver- sions the RAFT agent was still present in significant amounts. GPC analysis of Boc- $\mathbf{P}_{\mathbf{x}}$ isolated after $t_{\text {dead }}$ indicated that no chain extension had occurred, instead showing a bimodal distribution of molecular mass and high dispersities (Fig. S6B $\dagger$ ) demonstrating that after $t_{\text {dead }}$ the RAFT group had been degraded. To probe if the RAFT agent degradation was temperature driven, we isolated and purified a second "living" Boc$\mathbf{P}_{\mathbf{x}}$ at intermediate conversions $(\rho=52 \% t=30 \mathrm{~min}$ ) (Fig. S7 $\dagger$ ). This polymer was then heated for 90 minutes under standard polymerisation conditions, but this time without addition of $\mathbf{1}$ and initiator. We anticipated that heating the polymer this way should result in degradation of the RAFT agent, a hypothesis that was confirmed upon attempting to chain extend this terminated Boc- $\mathbf{P}_{\mathbf{x}}$. In this case, high dispersities, together with a shoulder at high molecular mass, were observed, indicating that the Boc- $\mathbf{P}_{\mathbf{x}}$ which had been subjected to further heating was "dead" (Fig. S7†). Additional evidence of the RAFT agent degradation was obtained from NMR spectroscopy, where the protons associated with both the $\mathrm{R}$ and $\mathrm{Z}$ end group of the polymer chain could be observed for the "living" Boc- $\mathbf{P}_{\mathbf{x}}$ whereas "dead" Boc- $\mathbf{P}_{\mathbf{x}}$ showed a loss of the $\mathrm{Z}$ group (Fig. S8 $\dagger$ ).

Seeing how running the polymerisations at $65^{\circ} \mathrm{C}$ gave the highest conversions $(90 \%)$ at $t_{\text {dead }}$ of all the conditions evaluated, we decided to target different degrees of polymerisation using these conditions (Fig. 4). As before, targeting higher DPs resulted in slower rates of polymerisation, in particular for DP200 and DP300. While slower rates had a significant effect on the maximum conversion achieved (approx. 90\%, 89\%, $68 \%$ and 55\% for DP 50, 100, 200 and 300 respectively), little effect was observed on the $t_{\text {dead }}$, with most polymerisations "stopping" after $1 \mathrm{~h}$ (Fig. 4A).

Under these optimised conditions, the polymerisations retained features of a controlled polymerisation, with the molecular mass of the polymers increasing linearly with conversion, narrow dispersities in molar mass (Fig. 4C) and good end group fidelity if isolated before $t_{\text {dead }}$. In all cases, the dispersities obtained were similar or lower to those reported previously. ${ }^{14}$ This improvement was particularly the case when targeting DPs of 100 and 200 with dispersities of $<1.4$ being observed at maximum conversion.
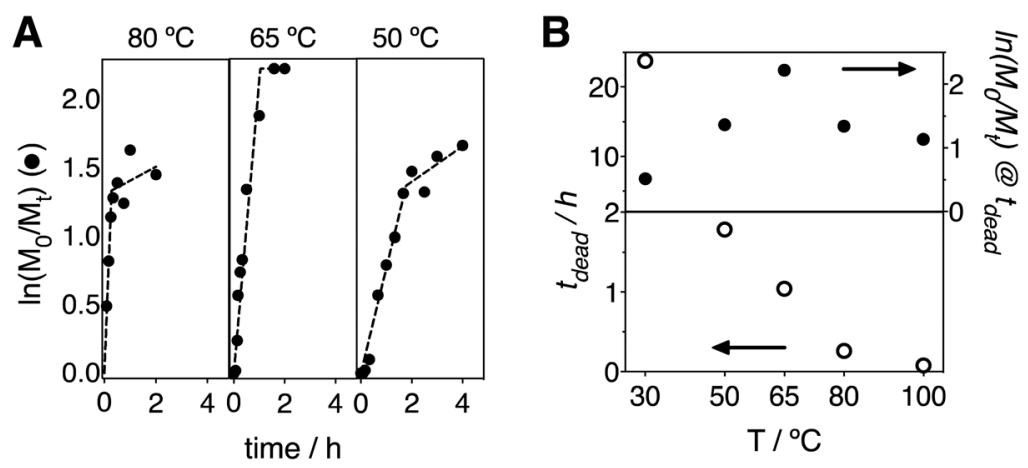

Fig. 3 (A) Plot of fractional concentration of monomer $\ln \left(M_{0} / M_{t}\right)$ vs. time for polymerisations of $N^{\prime}$-(tert-butoxycarbonyl)acryloyl hydrazide (1) performed at different temperatures. (B) Effect of temperature on the time at which deviation from linearity for the plot of $\ln [\mathrm{M}]_{0} /[\mathrm{M}]_{\mathrm{t}} v s$. time is observed $\left(t_{\text {dead }}\right)(O)$, and the fractional concentration of monomer $\ln \left(M_{0} / M_{t}\right)$ at this point $(\bullet)$. Conditions: $[M]=0.9 M,[M] /[C T A] /[V A-044]=50 / 1 / 0.2$. 

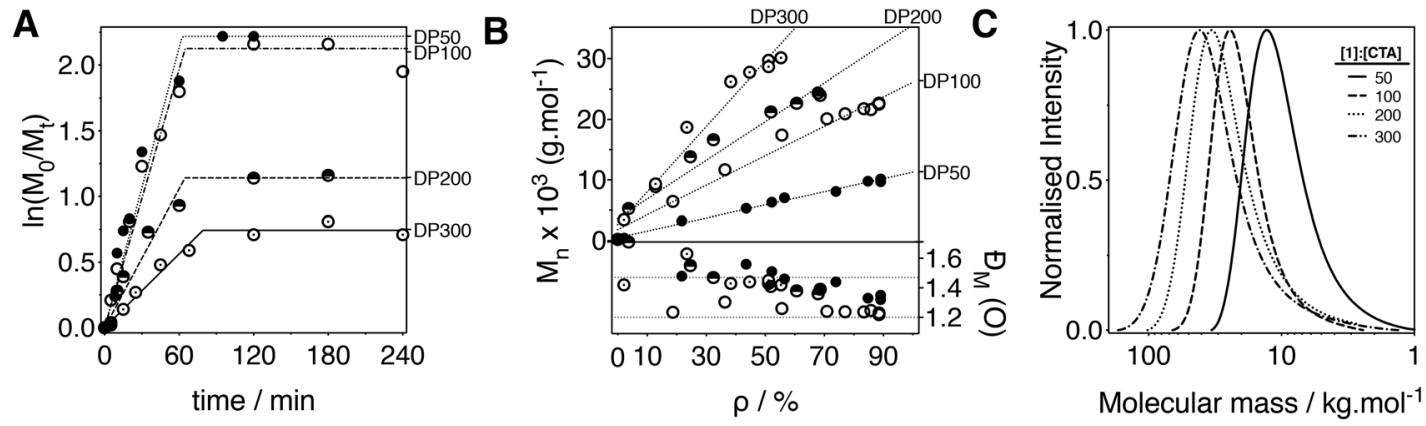

Fig. 4 (A) Plot of fractional concentration of monomer $\ln \left(M_{0} / M_{t}\right)$ vs. time. (B) Measured number average molecular mass $\left(M_{n}\right)$ vs. conversion $(\rho)$ (top) and dispersity in molecular mass $\left(\Theta_{M}\right)$ vs. conversion $(\rho)$ (bottom), for polymerisations of $N^{\prime}$-(tert-butoxycarbonyl)acryloyl hydrazide (1) performed at $65^{\circ} \mathrm{C}$ with different 1 : CTA ratios. (C) GPC chromatograms of the resulting polymers at the highest $\rho$ obtained. Conditions: [M] $=0.9 \mathrm{M}$, $[\mathrm{CTA}] /[\mathrm{VA}-044]=5 / 1 . \mathrm{M}_{\mathrm{n}}$ and $\Xi_{\mathrm{M}}$ calculated by GPC using $0.05 \mathrm{M} \mathrm{LiBr}$ in dimethylformamide (DMF) at $60^{\circ} \mathrm{C}$.

\section{Conclusion}

Here we have demonstrated the role of temperature and RAFT agent degradation in the polymerisation of $N^{\prime}$-(tert-butoxycarbonyl)acryloyl hydrazide (1). Our results highlight that the polymerisation of this hydrazide monomer 1 via RAFT can be severely hampered by the degradation of the chain transfer agent and that, under some circumstances, this degradation cannot be eliminated but rather outperformed if the rate of polymerisation is tuned. We demonstrate that by using a lowtemperature initiator such as VA-044, optimal polymerisation conditions can be achieved at $65{ }^{\circ} \mathrm{C}$. This way, poly $\left(N^{\prime}\right.$-(tertbutoxycarbonyl)acryloyl hydrazide)s with high degrees of polymerisation could be obtained while still maintaining low dispersities. We believe that further improvement of the polymerisation could be achieved through the choice of RAFT agents such as pyrazole or quaternised pyridinium dithiocarbamates, ${ }^{37,38}$ the use of photopolymerisation, ${ }^{39}$ or the use of alternative controlled polymerisation techniques. Our efforts in these directions will be reported in due course.

\section{Author contributions}

PFT, OC and PA designed the work. OC, PA, GS and AR performed all the experimental work. OC, AR and PFT analysed the data. OC and PFT wrote the paper, with all other authors contributing to the final version of the manuscript.

\section{Conflicts of interest}

There are no conflicts of interest.

\section{Acknowledgements}

All authors thank the P. F. T. group for useful discussions. P. F. T. thanks the University of Birmingham for the John Evans Fellowship. O. C. and P. A. thank BBSRC's Midlands
Integrative Biosciences Training Partnership MIBTP (BB/ M01116X/1) for PhD scholarships and A. R. thanks the European Union's Horizon 2020 Research and Innovation Programme (Marie Sklodowska-Curie Grant Agreement No. 795082).

\section{References}

1 J. E. Gestwicki, C. W. Cairo, L. E. Strong, K. A. Oetjen and L. L. Kiessling, J. Am. Chem. Soc., 2002, 124, 14922-14933.

2 R. Duncan, Nat. Rev. Drug Discovery, 2003, 2, 347-360.

3 C. Zhu, L. Liu, Q. Yang, F. Lv and S. Wang, Chem. Rev., 2012, 112, 4687-4735.

4 J. Li, F. Yu, Y. Chen and D. Oupický, J. Controlled Release, 2015, 219, 369-382.

5 J. J. Green and J. H. Elisseeff, Nature, 2016, 540, 386-394.

6 A. Muñoz-Bonilla and M. Fernández-García, Prog. Polym. Sci., 2012, 37, 281-339.

7 E.-R. Kenawy, S. D. Worley and R. Broughton, Biomacromolecules, 2007, 8, 1359-1384.

8 D. W. Pack, A. S. Hoffman, S. Pun and P. S. Stayton, Nat. Rev. Drug Discovery, 2005, 4, 581-593.

9 H. N. Kim, Z. Guo, W. Zhu, J. Yoon and H. Tian, Chem. Soc. Rev., 2011, 40, 79-93.

10 S.-J. Richards, M. W. Jones, M. Hunaban, D. M. Haddleton and M. I. Gibson, Angew. Chem., Int. Ed., 2012, 51, 78127816.

11 Z. Cao, L. Mi, J. Mendiola, J. R. Ella Menye, L. Zhang, H. Xue and S. Jiang, Angew. Chem., Int. Ed., 2012, 51, 26022605.

12 Functional Polymers by Post-Polymerization Modification, ed. P. Théato and H.-A. Klok, Wiley-VCH Verlag GmbH \& Co. KGaA, Weinheim, Germany, 2012.

13 J. Collins, Z. Xiao, M. Müllner and L. A. Connal, Polym. Chem., 2016, 7, 3812-3826.

14 D. N. Crisan, O. Creese, R. Ball, J. L. Brioso, B. Martyn, J. Montenegro and F. Fernandez-Trillo, Polym. Chem., 2017, 1, 1392-1399.

15 D. K. Kölmel and E. T. Kool, Chem. Rev., 2017, 117, 1035810376. 
16 E. R. L. Brisson, Z. Xiao, L. Levin, G. V. Franks and L. A. Connal, Polym. Chem., 2016, 7, 1945-1952.

17 E. M. Muzammil, A. Khan and M. C. Stuparu, RSC Adv., 2017, 7, 55874-55884.

18 K. Godula and C. R. Bertozzi, J. Am. Chem. Soc., 2010, 132, 9963-9965.

19 A. Kumar, R. R. Ujjwal, A. Mittal, A. Bansal and U. Ojha, ACS Appl. Mater. Interfaces, 2014, 6, 1855-1865.

20 J. M. Priegue, D. N. Crisan, J. Martínez-Costas, J. R. Granja, F. Fernandez-Trillo and J. Montenegro, Angew. Chem., Int. Ed., 2016, 55, 7492-7495.

21 A. Kumar, R. R. Ujjwal, A. Mittal, A. Bansal and U. Ojha, ACS Appl. Mater. Interfaces, 2014, 6, 1855-1865.

22 J. M. Priegue, I. Lostalé-Seijo, D. Crisan, J. R. Granja, F. Fernandez-Trillo and J. Montenegro, Biomacromolecules, 2018, 19, 2638-2649.

23 M. Juanes, O. Creese, P. Fernández-Trillo and J. Montenegro, MedChemComm, 2019, 526, 351-357.

24 J. D. Moskowitz, B. A. Abel, C. L. McCormick and J. S. Wiggins, J. Polym. Sci., Part A: Polym. Chem., 2015, 54, 553-562.

25 B. A. Abel and C. L. McCormick, Macromolecules, 2016, 49, 465-474.

26 E. A. Hoff, B. A. Abel, C. A. Tretbar, C. L. McCormick and D. L. Patton, Polym. Chem., 2017, 8, 4978-4982.
27 B. A. Chalmers, A. Alzahrani, G. Hawkins and F. Aldabbagh, J. Polym. Sci., Part A: Polym. Chem., 2017, 55, 2123-2128.

28 S. Perrier, Macromolecules, 2017, 50, 7433-7447.

29 J. Skey and R. K. O'Reilly, Chem. Commun., 2008, 31, 4183.

30 G. Moad, Prog. Polym. Sci., 2019, 88, 130-188.

31 Y. Zhou, Z. Zhang, A. Postma and G. Moad, Polym. Chem., 2019, 10, 3284-3287.

32 G. Gody, T. Maschmeyer, P. B. Zetterlund and S. Perrier, Nat. Commun., 2013, 4, 2505.

33 G. Gody, T. Maschmeyer, P. B. Zetterlund and S. Perrier, Macromolecules, 2014, 47, 639-649.

34 G. Gody, R. Barbey, M. Danial and S. Perrier, Polym. Chem., 2015, 6, 1502-1511.

35 Y. Zhou, J. He, C. Li, L. Hong and Y. Yang, Macromolecules, 2011, 44, 8446-8457.

36 T. M. Legge, A. T. Slark and S. Perrier, J. Polym. Sci., Part A: Polym. Chem., 2006, 44, 6980-6987.

37 J. Gardiner, I. Martinez-Botella, J. Tsanaktsidis and G. Moad, Polym. Chem., 2016, 7, 481-492.

38 D. J. Keddie, C. Guerrero-Sanchez, G. Moad, E. Rizzardo and S. H. Thang, Macromolecules, 2011, 44, 67386745.

39 M. Chen, M. Zhong and J. A. Johnson, Chem. Rev., 2016, 116, 10167-10211. 